\title{
Mitogen-activated protein kinase phosphatase 1 is involved in tamoxifen resistance in MCF7 cells
}

\author{
GANG MA $^{1 *}$, YIXIA PAN ${ }^{2 *}$, CAN ZHOU $^{1}$, RUIFANG SUN ${ }^{3}$, JINGJING BAI $^{4}$, \\ PEIJUN LIU ${ }^{5}$, YU REN ${ }^{1}$ and JIANJUN HE ${ }^{1}$

\begin{abstract}
${ }^{1}$ Department of Breast Surgery, The First Affiliated Hospital, Medicine College of Xi'an Jiaotong University; of Xi'an Jiaotong University, Xi'an, Shaanxi 710061; ${ }^{4}$ Shaanxi Province People's Hospital, Xi'an, Shaanxi 710068;

${ }^{5}$ Department of Translational Medicine Center, The First Affiliated Hospital, Medicine College of Xi'an Jiaotong University, Xi'an, Shaanxi 710061, P.R. China
\end{abstract} \\ ${ }^{2}$ Department of Gynecology, Northwest Women and Childrens Hospital; ${ }^{3}$ Department of Pathology, Medicine College
}

Received May 30, 2015; Accepted July 8, 2015

DOI: 10.3892/or.2015.4244

\begin{abstract}
Tamoxifen resistance is a major clinical problem for ER-positive breast cancer, but the underlying mechanism is not completely elucidated. In the present study, we reported that mitogen-activated protein kinase (MAPK) phosphatase 1 (MKP-1), a member of the family of MKPs, is involved in tamoxifen resistance. We found that MKP1 expression increased in tamoxifen resistant MCF7 cells. To explore the possible role of MKP1 in tamoxifen resistance, siRNA targeting MKP1 was transfected into tamoxifen resistant MCF7 cells. To our surprise, knockdown of MKP-1 promoted cell death induced by tamoxifen. On the other hand, the MKP1 overexpressed MCF7 cell clone was established and MKP1 overexpression effectively attenuated MCF7 cell death induced by tamoxifen. In addition, we revealed that MKP1 inhibited tamoxifen-mediated JNK activation in tamoxifen resistant MCF7 and MCF7 cells, and by this mechanism MKP1 was able to inhibit tamoxifen-induced cell death. We also showed that combined appliaction of MKP1 inhibitor triptolide and tamoxifen can effectively increase tamoxifen sensitivity in tamoxifen resistant MCF7 cells. Collectively, our results indicated that MKP-1 can attenuate tamoxifen-induced cell death
\end{abstract}

Correspondence to: Professor Jianjun He, Department of Breast Surgery, The First Affiliated Hospital, Medicine College of Xi'an Jiaotong University, 277 Yanta Western Road, Xi'an, Shaanxi 710061, P.R. China

E-mail: chinahjj@163.com

*Contributed equally

Abbreviations: MKP1, mitogen-activated protein kinase phosphatase 1; MAPKs, mitogen-activated protein kinases; ER, estrogen receptor; JNK, JUN NH2-terminal kinases; TAM, 4-hydroxy tamoxifen

Key words: mitogen-activated protein kinase phosphatase 1, MCF7 cells, tamoxifen resistance, cell apoptosis, JUN NH2-terminal kinases through inhibiting the JNK signal pathway, which represents a novel mechanism of tamoxifen resistance in MCF7 cells.

\section{Introduction}

Breast cancer is classified into three basic subtypes including ER-positive subtype, HER2 amplified subtype and triplenegative breast cancer subtype (1). Approximately $75 \%$ breast cancers are classified into ER-positive subtype and need to be given endocrine treatment (2). Tamoxifen, a selective estrogen receptor modulator, has been used as a basic endocrine drug for the past four decades (3). However, the effect of tamoxifen is limited by occurrences of de novo and acquired resistance (4). In previous studies $(2,5)$, tamoxifen resistance has been attributed to many different reasons including: loss of $\mathrm{ER} \alpha$, signal pathways crosstalk, deregulation of coregulators, as well as altered expression of microRNAs. Even so, there is still a great need to explore the underlying mechanism of tamoxifen resistance.

The signal pathway of mitogen activated protein kinases (MAPKs) has been shown to be involved in cancer development and progression (6). Studies have also supported that the MAPKs are implicated in tamoxifen resistance. Activated ERK has been shown to phosphorylate ER $\alpha$, leading to ligand-independent activation of ER signal and tamoxifen resistance (7). High level of phosphorylated p38 has been observed in tamoxifen treated breast cancer specimens and activation of p-38 has been proven to induce tamoxifen resistance through activation of ER (8). Studies have also shown that AP1, a downstream target of ERK and JNK, promotes transcription in tamoxifen-treated breast cancer (9). In addition, JNK has been shown to contribute to tamoxifen pharmacological action through mediating apoptosis (10).

The MAPK phosphatases (MKPs), which can specifically bind and dephosphorylate MAPKs, are negative regulators of MAPKs (11). MKP1 is the first identified member of MKPs family and thus frequently studied. MKP1 has been shown to dephosphorylate all three members of MAPKs, particularly p38 and JNK (12), in inflammatory response and metabolic homeostasis $(13,14)$. MKP1 also plays an important role in 
cancer (15). Li et al (16) reported that MKP1 is a transcriptional target of tumor suppressor p53, and thus involved in the negative regulation of cell proliferation. Several studies have also shown that MKP1 can attenuate chemotherapyinduced apoptosis in cancers through dephosphorylation of JNK (17-20).

Herein, we show that MKP1 expression was increased in tamoxifen resistant MCF7 cells. When using MKP1 siRNA knocked down MKP1 in tamoxifen resistant MCF7 cells, tamoxifen-mediated cell death increased. On the contrary, overexpression of MKP1 in MCF7 cells attenuated tamoxifen-mediated cell death. In addition, change of MKP1 expression was correlated with tamoxifen-induced activation of JNK in MCF7 and tamoxifen resistant MCF7 cells. Importantly, using JNK inhibitor SP600125, we showed that the inhibition effect of MKP1 on tamoxifen-mediated cell death is through blocking JNK activation.

\section{Material and methods}

Reagents and antibodies. Tissue culture media and materials were purchased from HyClone. Charcoal stripped fetal bovine serum (cs-FBS) and fetal bovine serum (FBS) were from PAA (A-15-119). 4-Hydroxy tamoxifen (TAM) was from Sigma and freshly dissolved in ethanol at stock concentration $10 \mathrm{mmol} / \mathrm{l}$. The TurboFect transfection reagent was from Thermo Scientific (R0531). Cell Counting Kit-8 (CCK-8) was from Dojin Laboratories of Kumamoto (CK04-01). Cell apoptosis detection kit was from Beyotime (C1052). The cDNA synthesis and real-time PCR reagents were purchased from Takara (D6110A). MKP1 siRNA pool (UGGAGCAUAUCGUGCC GAA and AAGAUAUGCUCGACGCCUU); and scrambled siRNA (CGTACGCGGAATACTTCGA) were purchased from Dharmacon. The empty vector pCMV-Tag-2A and mkp1-pCMV-Tag-2A plasmids were kind gifts from Professor Lianfeng Zhang. The SP600125 (sc-200635), triptolide (sc-200122) and dexamethasone were from Santa Cruz Biotechnology. The following antibodies were used in the present study: anti-MKP-1 (sc-370; Santa Cruz); anti-MAPKs (cst-9926; Cell Signaling Technology), anti-p-MAPKs (cst-9938), anti p-c-jun (cst-9164) and anti-PARP (cst-9532); anti- $\beta$-actin (A-5316; Sigma). Caspase 3/7 activity assay kit was from Beyotime.

Cell lines and treatment. Human breast cancer MCF7 cell line was purchased from the Cell Bank of Chinese Academy of Science. Tamoxifen resistant MCF7 cell line LCC2 (21) was a kind gift from Professor Xinyi Chen. MCF7 cells were maintained in Dulbecco's modified Eagle's medium (DMEM) containing $10 \%$ FBS supplemented with $2 \mu \mathrm{M} \mathrm{L}$-glutamine, $50 \mathrm{U} / \mathrm{ml}$ penicillin and $50 \mu \mathrm{g} / \mathrm{ml}$ streptomycin. LCC2 cells were maintained in phenol red free DMEM containing $10 \%$ cs-FBS supplemented with $2 \mu \mathrm{M}$ L-glutamine, 5,050 U/ml penicillin and $50 \mu \mathrm{g} / \mathrm{ml}$ streptomycin. High-dose tamoxifen resistant MCF7 cells (MCF7-TR) were derived from LCC2 cells based on the following strategy: LCC 2 cells were treated with gradually increased concentration of TAM (1-5 $\mu \mathrm{M})$ for two months, and then were kept in medium containing $5 \mu \mathrm{M}$ TAM for 6 months. All of the cells were incubated at $37^{\circ} \mathrm{C}$ in $5 \% \mathrm{CO}_{2}$. Prior to experiments, all of the cells were cultured in phenol red free DMEM containing 5\% cs-FBS for one week.

MTT analysis. The cell viability was determined by CCK-8 assay according to the manufacturer's instruction. Cells were incubated with $10 \mu \mathrm{l} \mathrm{CCK}-8$ for $3 \mathrm{~h}$ at $37^{\circ} \mathrm{C}$. The absorbance was measured at $450 \mathrm{~nm}$. The viability of cells was calculated as: (OD450 nm of sample/OD450 nm of control) x 100\%. All experiments were performed in triplicate.

Flow cytometric analysis of cell apoptosis. Equal numbers of cells $\left(2 \times 10^{6}\right)$ were seeded into $60 \mathrm{~mm}$ dishes and incubated at $37^{\circ} \mathrm{C}$ in $5 \% \mathrm{CO}_{2}$. Overnight, cells were treated with different doses of TAM for $48 \mathrm{~h}$. Both floating and adherent cells were collected by trypsin/EDTA-free, washed in cold PBS and centrifuged at $1,000 \mathrm{rpm}$ for $5 \mathrm{~min}$. The supernatant was discarded. Binding buffer $(500 \mu \mathrm{l})$ was added to the deposit which was resuspended, then $5 \mu \mathrm{l}$ Annexin V-FITC and $5 \mu \mathrm{l}$ propidium iodide were added. After incubated at RT for 15 min, cells were analyzed by FACScan flow cytometer. For each sample, 10,000 events were analyzed.

cDNA synthesis and quantitative RT-PCR analysis. Cell monolayers were lysed and total RNA was isolated using TRIzol reagent according to the manufacturer's protocol. Total RNA (100 ng) was reverse transcribed using Takara PrimeScript First Strand cDNA Synthesis kit. Real-time PCR was performed using the Takara SYBR Premix Ex Taq kit. Primers used to amplify MKP1 and $18 \mathrm{~S}$ were as follows: MKP1F, 5'-gggacgcgcggtgaag-3' and MKP1R, 5'-gatcttgtgcggttttttgtgg-3'; 18SF, 5'-tctcaaagattcgccatgc-3' and 18SR, 5'-tcaccaacggagaccttg-3'.

MKP1 silencing with small interfering RNA. MCF7-TR cells were transiently transfected with MKP1 siRNA and scrambled siRNA into 6-well plates with TurboFect reagent according to the manufacturer's instruction. Forty-eight hours after transfection, cells were used for the experiments. Cells transfected with scrambled siRNA were used as control.

Establishment of MKPI overexpressing MCF7 cells. MCF7 cells were transfected with either the empty vector pCMV-Tag-2A plasmid or mkp1-pCMV-Tag-2A plasmid using TurboFect reagent according to the instruction of TurboFect reagent. Transfected cells were selected with $400 \mu \mathrm{g} / \mathrm{ml} \mathrm{G} 418$ (Sigma) for 4 weeks. Single clones were picked up for establishing stable clones. MKP-1 expression was determined by western blotting.

Western blot analysis. Western blot analysis in cultured cells was carried out as previously reported (22). Briefly, cells were washed in PBS and scraped, and whole-cell lysates were prepared and centrifuged at $12,000 \mathrm{rpm}$ for $20 \mathrm{~min}$ at $4^{\circ} \mathrm{C}$. Equal amounts of total protein $(40 \mu \mathrm{g})$ were loaded on $10 \%$ SDS-PAGE gels and then transferred onto a polyvinylidene fluoride membrane with a Semi-Dry Transfer Cell. Membranes were blocked in TBS-Tween $(0.1 \%) / 5 \%$ milk and were then hybridized with primary antibody overnight at $4^{\circ} \mathrm{C}$. Membranes were incubated with the secondary antibody for $1 \mathrm{~h}$ at RT. Resulting bands were visualized using enhanced 



Figure 1. (A-C) MCF7, LCC2 and MCF7-TR cells were treated with control medium, estrogen (E2; $50 \mathrm{nM}$ ) and tamoxifen (TAM; 1 and $5 \mu \mathrm{M})$ for 7 days. Cell proliferation was measured using CCK-8 assay. The growth curves were representative of three independent experiments. (D) Relative MKP1 mRNA level was measured by quantitative RT-PCR in MCF7, LCC2 and MCF7-TR cells. MKP1 mRNA expression value was relative to the level of 18S mRNA. Significant difference "P<0.05 (mean $\pm \mathrm{SD}, \mathrm{n}=3$ ). (E) MKP1 protein level was detected by western blotting in MCF7, LCC2 and MCF7-TR cells which were treated with control medium, E2 $(50 \mathrm{nM})$ and TAM $(5 \mu \mathrm{M})$ for $24 \mathrm{~h}, \beta$-actin was used as loading control.

chemiluminescence reagents (Millipore). Images were scanned and quantified by ChemiDoc ${ }^{\mathrm{TM}}$ MP System (Bio-Rad).

Determination of caspase 3/7 activity. Caspase 3/7 activity was detected using the Caspase 3/7 Activity Assay kit following the protocol provided by the manufacturer. Cells were harvested and then incubated with Ac-DEVD-Pna for $2 \mathrm{~h}$ at $37^{\circ} \mathrm{C}$. The absorbance was measured at $405 \mathrm{~nm}$. The caspase 3/7 activity of the cells was calculated as: (OD405 nm of sample/OD405 $\mathrm{nm}$ of control) x 100\%. All experiments were performed in triplicate.

Statistical analysis. Statistical analysis was carried out with Statistical Package for the Social Sciences version 19.0 (SPSS, Inc.). Statistical significance $(\mathrm{p}<0.05)$ between experimental and control groups was calculated by LSD-t test. All data are expressed as the means \pm SD for the number of experiments.

\section{Results}

MKPI expression was upregulated in tamoxifen resistant breast cancer MCF7 cells. The LCC2 cells were derived from MCF7 cells and have been used as a tamoxifen resistant cell model for several years (21). In the present study, we established a high-dose tamoxifen resistant MCF7 cell line (MCF7-TR) from LCC2 cells by treating LCC2 with $5 \mu \mathrm{M}$ TAM for $>6$ months. In order to verify resistant character of the MCF7-TR cells, we treated parental MCF7, LCC2 and MCF7-TR cells with estrogen (E2), tamoxifen (TAM) and control medium for 7 days. The cell proliferation was detected by MTT test and cell growth curves were depicted. As shown in Fig. 1A-C, MCF7-TR cells were resistant to high-dose
(5 $\mu \mathrm{M}$ ) tamoxifen, whereas MCF7 and LCC2 cells were not. For exploring the possible role of MKP1 in tamoxifen resistance, we examined mRNA level of MKP1 in all three cell lines using quantitative RT-PCR. The results indicated that MKP1 mRNA level was increased in MCF7-TR cells compared with MCF7 and LCC2 cells (Fig. 1D). Consistent with MKP1 mRNA, MKP1 protein expression was also increased in MCF7-TR cells (Fig. 1E). Taken together, all the above results suggest that MKP-1 expression is upregulated in high-dose tamoxifen resistant MCF7 cells.

MKP1 inhibits tamoxifen-mediated apoptosis in MCF7-TR and MCF7 cells. As MKP1 expression increased in MCF7-TR cells compared with MCF7 and LCC2 cells, we asked whether MKP1 is correlated with tamoxifen resistance. To this end, we transiently transfected scrambled siRNA and MKP1 siRNA to MCF7-TR cells. The knockdown efficiency was determined by western blotting and the result is shown in Fig. 2A. We then asked whether MKP1 plays a role in tamoxifen-induced apoptosis (23). To this end, both scrambled siRNA and MKP1 siRNA transfected MCF7-TR cells were treated with gradually increased micromolar concentration of tamoxifen $(0-10 \mu \mathrm{M})$ for $48 \mathrm{~h}$. Apoptosis was determined by flow cytometry. As shown in the Fig. $2 \mathrm{~B}$ and C, tamoxifeninduced apoptosis was sharply increased in MKP1 siRNA transfected MCF7-TR cells compared to scrambled siRNA transfected MCF7-TR cells, indicating that knockdown of MKP1 enhanced tamoxifen sensitivity of MCF7-TR cells. On the other hand, we established MKP1 stably overexpressing MCF7 cell clones by transfection with mkp1-pCMV-Tag-2A plasmid. The cell clones were verified by western blotting (Fig. 2D). We also detected the apoptosis induced by 
A

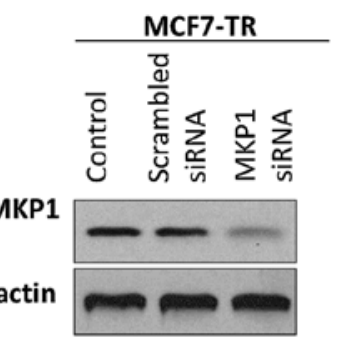

B

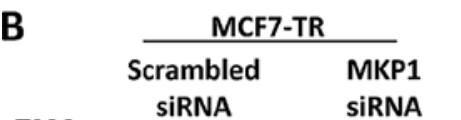

C

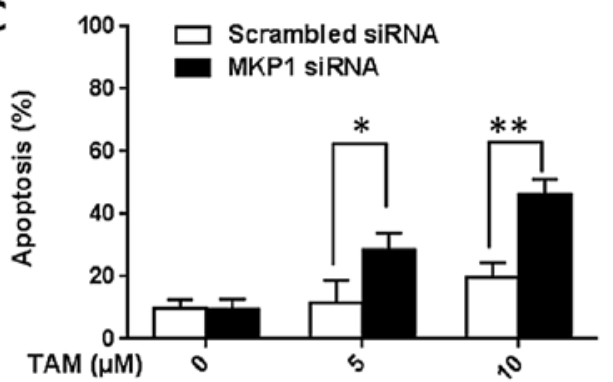

D

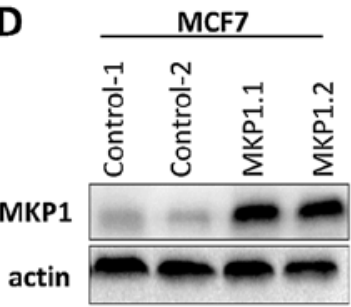

E
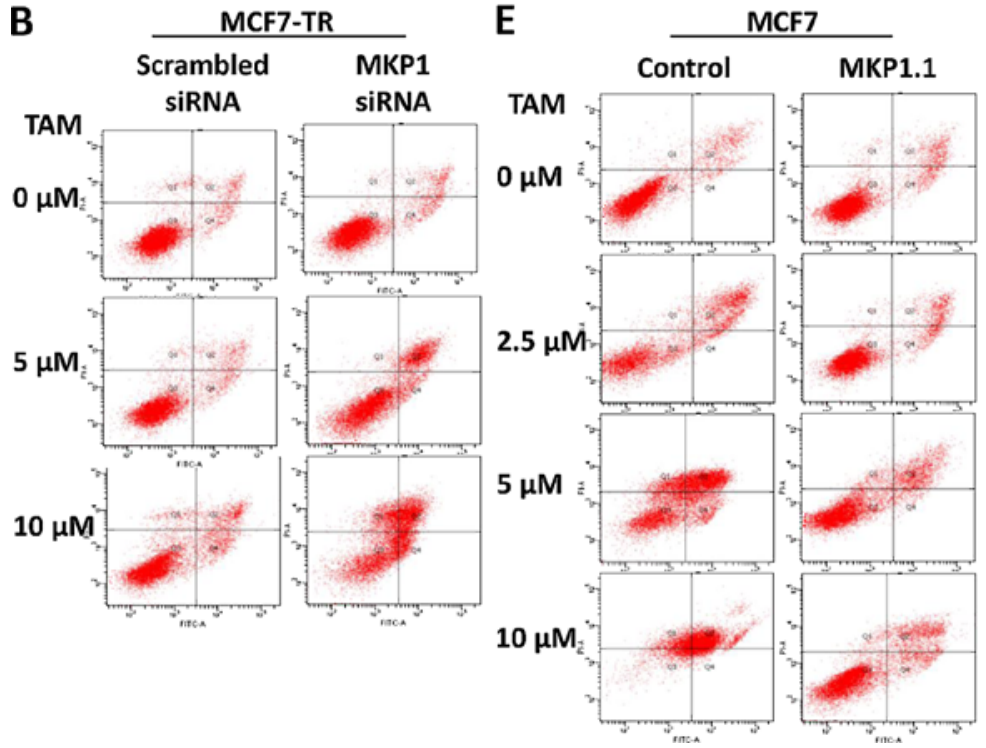

$\mathbf{F}$

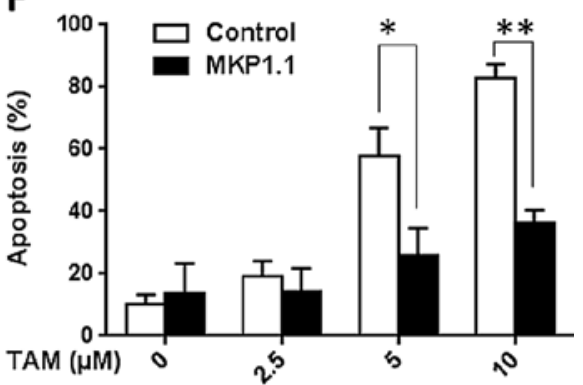

Figure 2. (A) MKP1 expression was detected in MCF7-TR cells transfected with scrambled siRNA and MKP1 siRNA. (B) Flow cytometry which measured apoptosis of scrambled siRNA and MKP1 siRNA transfected MCF7-TR cells after TAM treated for $48 \mathrm{~h}$. (C) Comparison of apoptosis rate between scrambled siRNA and MKP1 siRNA transfected MCF7-TR cells. Significant difference ${ }^{*} \mathrm{P}<0.05 ;{ }^{* *} \mathrm{P}<0.01$ (mean $\pm \mathrm{SD}$, $\mathrm{n}=3$ ). (D) MKP1 expression was detected in control MCF7 cells and MKP1 overexpressed MCF7 cells. (E) Flow cytometry which measured apoptosis of MCF7/control and MCF7/MKP1.1 cells after TAM treated for $48 \mathrm{~h}$. (F) Comparison of apoptosis rate between MCF7/control and MCF7/MKP1.1 cells. Significant difference * $<<0.05$; ${ }^{* *} \mathrm{P}<0.01$ (mean $\pm \mathrm{SD}, \mathrm{n}=3$ ).

tamoxifen $(0-10 \mu \mathrm{M})$ in MKP1 overexpressed MCF7 cells (MCF7/MKP1.1) and empty vector plasmid transfected MCF7 cells (MCF7/control). As shown in Fig. 2E and F, results of flow cytometry indicated that overexpression of MKP1 effectively inhibits tamoxifen-induced apoptosis in MCF7 cells. Taken together, the above results suggest that MKP1 can protect MCF7 and MCF7-TR cells from tamoxifeninduced apoptosis.

MKP1 blocks TAM-mediated activation of MAPKs. Since MKP1 is a phosphatase which specifically targets activated MAPKs (11), we hypothesized that knockdown of MKP1 influences the activation of MAPKs in tamoxifen-treated MCF7-TR cells. The phosphorylated MAPKs and total MAPKs were analyzed by western blotting in scrambled siRNA and MKP1 siRNA transfected MCF7-TR cells which were treated with tamoxifen for 24 and $48 \mathrm{~h}$ (Fig. 3A and B). As shown in the relative quantitation histograms of western blotting bands (Fig. 3D and E), the increased level of phosphorylated ERK1/2 and p-38 were comparable between scrambled siRNA transfected MCF7-TR cells and MKP1 siRNA transfected MCF7-TR cells after tamoxifen treatment. Interestingly, the expression of phosphorylated JNK in MKP1 siRNA transfected MCF7-TR cells was sharply increased compared to expression of phosphorylated JNK in scrambled
siRNA transfected MCF7-TR cells after tamoxifen treatment (Fig. 3F), indicating that knockdown of MKP1 discharges the inhibition on tamoxifen-induced activation of JNK in MCF7-TR cells. Tamoxifen has been shown to activate several signaling pathways which were upstream of MAPKs and then induce activation of MAPKs in MCF7 cells $(23,24)$. We next wanted to know whether overexpression of MKP1 affects the tamoxifen-induced activation of MAPKs in MCF7 cells. The phosphorylated MAPKs and total MAPKs were determined by western blotting in MCF7/control and MCF7/MKP1.1 cells after tamoxifen treatment for 24 and $48 \mathrm{~h}$ (Fig. 4A and B). As shown in the relative quantitation histograms of western blotting bands (Fig. 4D-F), tamoxifen-induced phosphorylation of MAPKs was decreased in MCF7/MKP1.1 cells compared to MCF7/control cells (Fig. 4D-F), indicating that overexpression of MKP1 in MCF7 attenuates the tamoxifen-induced activation of MAPKs. Taken together, these results suggest that MKP1 can negatively regulate tamoxifen-induced activation of MAPKs, especially JNK, in MCF7-TR and MCF7 cells.

MKP1 inhibits tamoxifen-induced apoptosis through blocking $J N K$ signaling. It has been shown that activation of JNK contributes to tamoxifen-mediated apoptosis $(10,25,26)$. Our results showed that MKP1 inhibited tamoxifen-induced apoptosis and JNK activation in MCF7-TR and MCF7 cells. Hence, 
A
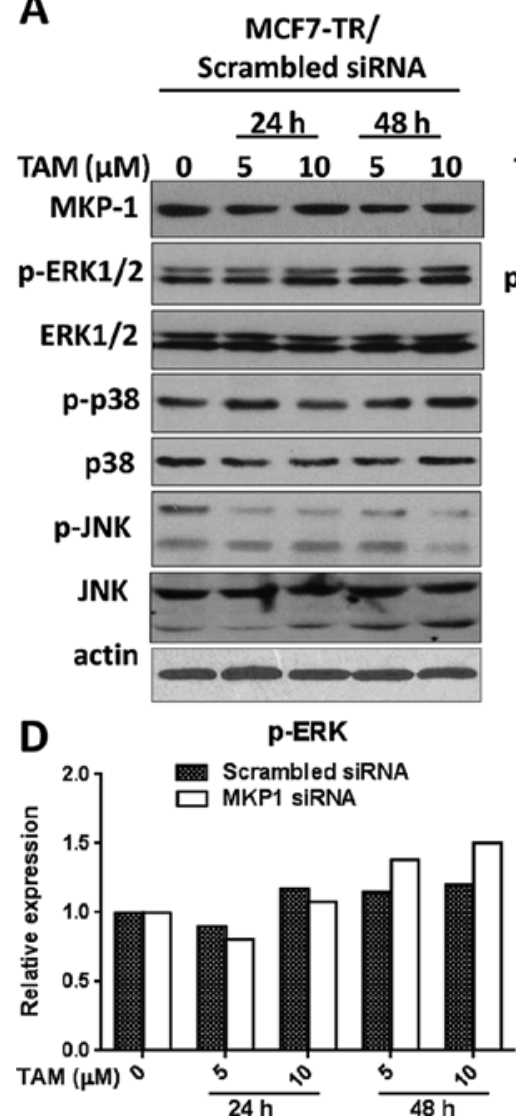

B

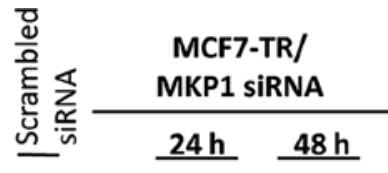

$\begin{array}{lllllll}\operatorname{TAM}(\mu \mathrm{M}) & 0 & 0 & 5 & 10 & 5 & 10\end{array}$
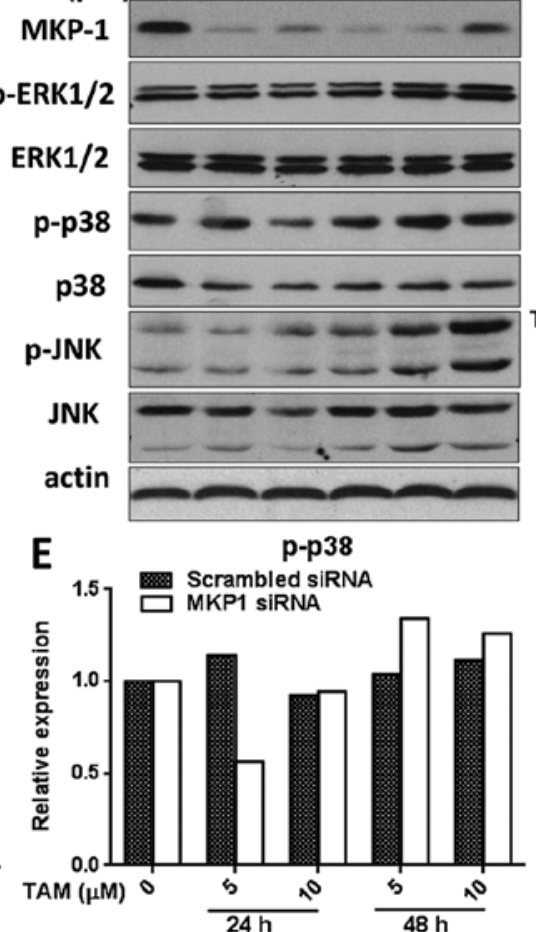

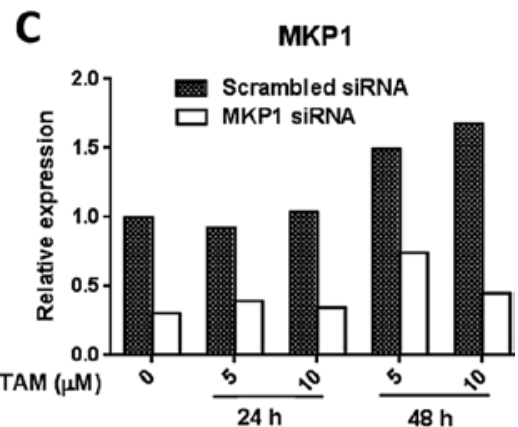

F

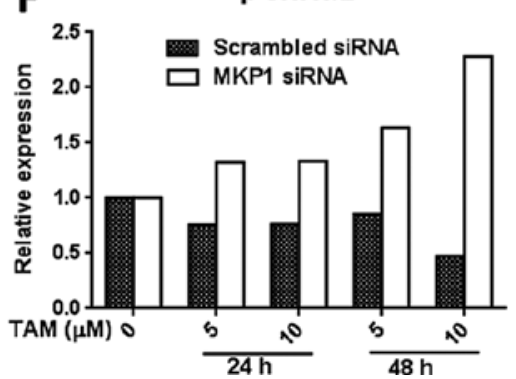

Figure 3. (A) Western blotting of MKP1, p-MAPKs and total MAPKs in scrambled siRNA transfected MCF7-TR cells after TAM treatment for 24 and $48 \mathrm{~h}$. (B) Western blotting of MKP1, p-MAPKs and total MAPKs in MKP1 siRNA transfected MCF7-TR cells after TAM treatment for 24 and $48 \mathrm{~h}$. (C) Histograms represent the relative quantitative comparison of MKP1 expression between A and B. (D-F) Histograms represent the relative quantitative comparison of p-MAPKs level between A and B.

A

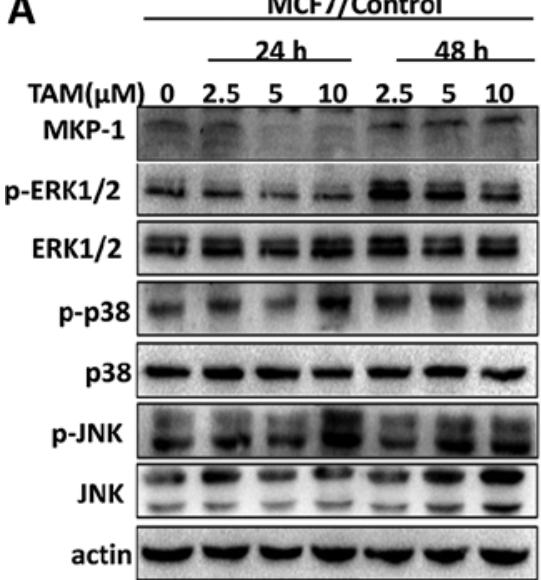

D

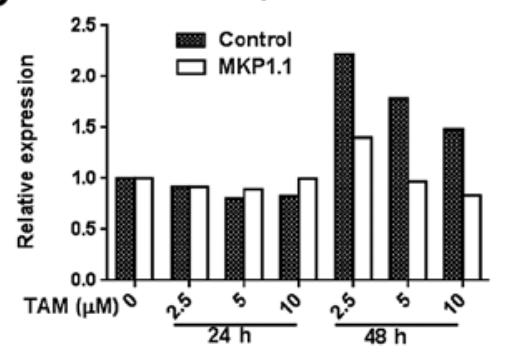

B

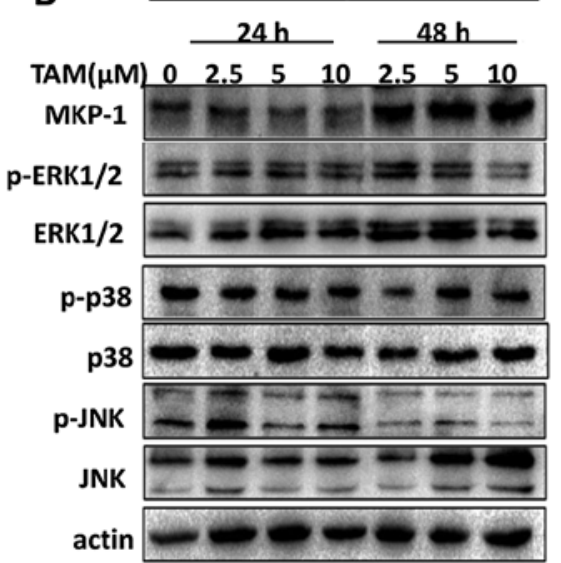

$\mathbf{E}$



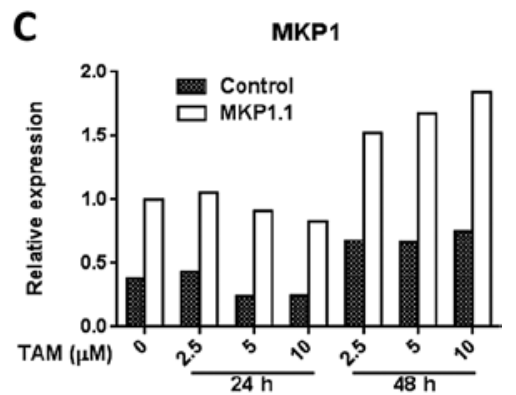

$\mathbf{F}$

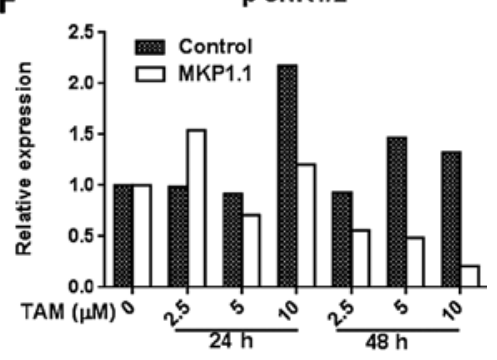

Figure 4. (A) Western blotting of MKP1, p-MAPKs and total MAPKs in MCF7/control cells after TAM-treated for 24 and $48 \mathrm{~h}$. (B) Western blotting of MKP1, p-MAPKs and total MAPKs in MCF7/MKP1.1 cells after TAM treatment for 24 and $48 \mathrm{~h}$. (C) Histograms represent the relative quantitative comparison of MKP1 expression between A and B. (D-F) Histograms represent the relative quantitative comparison of p-MAPKs level between A and B. 

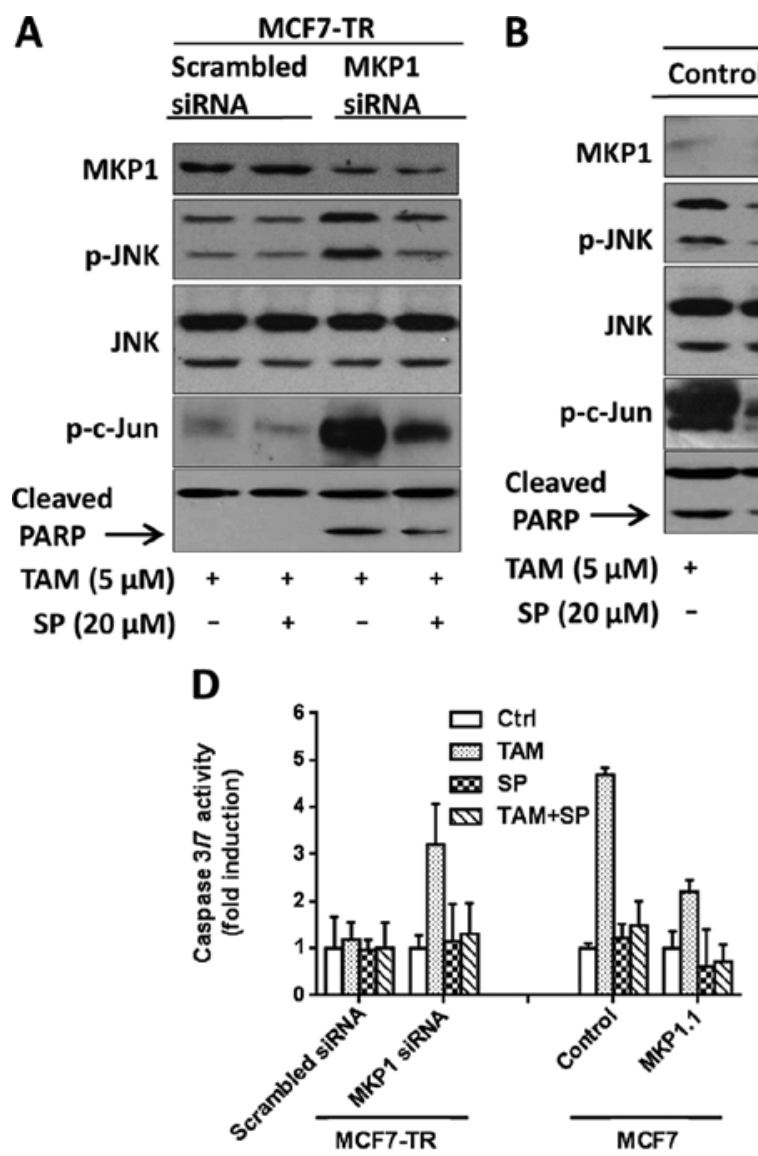


Figure 5. (A) Expression of MKP1, p-JNK, JNK, p-c-jun and cleaved PARP were determined by western blotting in scrambled siRNA and MKP1 siRNA transfected MCF7-TR cells, which were treated with either $5 \mu \mathrm{M}$ TAM for $48 \mathrm{~h}$ or $20 \mu \mathrm{M} \mathrm{SP} 600125$ (SP) for $3 \mathrm{~h}$ and then $5 \mu \mathrm{M}$ TAM for $48 \mathrm{~h}$. (B) Expression of MKP1, p-JNK, JNK, p-c-jun and cleaved PARP were determined by western blotting in MCF7/control and MCF7/MKP1.1 cells, which were treated with either $5 \mu \mathrm{M}$ TAM for $48 \mathrm{~h}$ or $20 \mu \mathrm{M} \mathrm{SP} 600125$ (SP) for $3 \mathrm{~h}$ and then $5 \mu \mathrm{M}$ TAM for $48 \mathrm{~h}$. (C) Histograms represent the apoptosis rate of cells in A and B. Significant difference ${ }^{*} \mathrm{P}<0.05 ;{ }^{* *} \mathrm{P}<0.01$ (mean $\pm \mathrm{SD}, \mathrm{n}=3$ ). (D) Histograms represent caspase $3 / 7$ activity of cells in A and B. (E) MCF7-TR cells were treated with vehicle (-), $5 \mu \mathrm{M}$ TAM, $1 \mu \mathrm{M}$ triptolide or both of compounds for $48 \mathrm{~h}$. Western blot analyses were performed using MKP1, p-JNK, JNK, p-c-jun and PARP antibodies.

we asked whether MKP1 mediates inhibition of tamoxifeninduced apoptosis is through blocking JNK activation. To this end, we used JNK inhibitor SP600125 (SP) to inhibit the tamoxifen-induced JNK activation in MKP1 siRNA transfected MCF7-TR and MCF7 cells. All cells were treated with the following protocol: incubated with $20 \mu \mathrm{M}$ SP600125 for $3 \mathrm{~h}$ and then treated with tamoxifen for $48 \mathrm{~h}$ or only treated with tamoxifen for $48 \mathrm{~h}$. After that, cells were harvested and expression of MKP1, p-JNK, JNK, p-c-jun and cleaved PARP were determined by western blotting (Fig. 5A and B). As shown in Fig. 5A, SP600125 effectively inhibited tamoxifenmediated JNK activation and cleaved PARP in MKP1 siRNA transfected MCF7-TR cells. Tamoxifen-induced apoptosis in MKP1 siRNA transfected MCF7-TR cells was sharply reduced (Fig. 5C). On the other hand, SP600125 also inhibited tamoxifen-mediated JNK activation in MCF7 cells and protected MCF7 cells from tamoxifen-induced apoptosis (Fig. 5B and C). We also analyzed the caspase 3/7 activity of cells after treatment with tamoxifen alone or tamoxifen in combination with SP600125. As shown in Fig. 5D, SP600125 treatment attenuated TAM-induced caspase 3/7 activity in MKP1 siRNA-transfected MCF7-TR cells.
Taken together, these results suggest that JNK signaling is involved in TAM-induced apoptosis and MKP1 inhibition of TAM-induced apoptosis is through blocking JNK activation.

Triptolide has been shown to inhibit MKP1 expression $(27,28)$. In the present study, we combined triptolide with tamoxifen to treat MCF7-TR cells. As shown in Fig. 5E, triptolide reduced MKP1 expression in MCF7-TR cells. Moreover, combined application of triptolide and tamoxifen sharply enhanced tamoxifen-induced activation of JNK and cleaved PARP compared with tamoxifen treatment alone, indicating that MKP1 inhibitor may be a new therapeutic strategy for tamoxifen resistance.

\section{Discussion}

Tamoxifen has been shown to induce both cytostatic (proliferation arrest) and cytotoxic effect (cell death or apoptosis) through ER $\alpha$-dependent and -independent pathways $(23,24)$. In MCF7 cells, the antitumor effects of tamoxifen through $\mathrm{ER} \alpha$ and non-ER $\alpha$ signal pathway were dependent on concentration of tamoxifen (nanomolar or micromolar) $(23,29)$. In other words, when used at nanomolar concentration, such as 
$100 \mathrm{nM}$, tamoxifen mainly induced cell growth arrest (cytostatic effects) through inhibition of ER signaling, whereas used at micromolar concentration, such as $5 \mu \mathrm{M}$, tamoxifen mainly induced cell death (cytotoxic effect) through ER-independent signal pathways. In the present study, we established a highdose TAM resistant MCF7 cell line (MCF7-TR) from the verified tamoxifen resistant LCC2 cells (21). MCF7-TR cells are not only resistant to nanomolar (low-dose) tamoxifeninduced cell growth arrest, but also resistant to micromolar (high-dose) tamoxifen-induced cell death. We detected the MKP1 expression in MCF7-TR cells and MCF7 cells, and found that both of MKP1 mRNA and protein expression increased in MCF7-TR cells. Several studies have shown that MKP1 increases in poorly differentiated and late stage of breast cancer but not in early stage of breast cancer $(30,31)$. Our data indicated that MKP1 expression is higher in tamoxifen resistant MCF7-TR cells compared with parental MCF7 cells, which is consistent with the previous studies.

MKP1 has been shown to induce drug resistance through inhibiting apoptosis (18-20,32). In the present study, knockdown of MKP1 in MCF7-TR cells increased cell death induced by micromolar concentration of tamoxifen. On the other hand, overexpression of MKP1 in MCF7 cells protected cells from cytotoxic effect mediated by micromolar concentration of tamoxifen. These results showed that MKP1 expression in MCF7-TR and MCF7 cells protects cell from tamoxifeninduced cytotoxic effect, indicating that MKP1 may be involved in tamoxifen resistance of MCF7 cells.

It has been reported that tamoxifen induces activation of MAPKs in breast cancer $(33,34)$. In the present study, detection of phosphorylated MAPKs in MKP1 knockdown MCF7-TR and MCF7 cells after tamoxifen treatment showed similar results (as shown in Figs. 3B and 4A). Since MKP1 specifically and negatively regulated MAPKs, we explored whether MKP1 inhibits tamoxifen-induced activation of JNK in MCF7-TR and MCF7 cells. As shown in Figs. 3A and 4B, the MKP1 highly-expressed MCF7-TR and MCF7 cells exhibited declining phosphorylation of MAPKs, especially phosphorylation of JNK, after tamoxifen treatment, indicating that MKP1 expressed in MCF7-TR and MCF7 cells inhibits the activation of MAPKs.

It has been reported that activation of JNK contributes to tamoxifen cytotoxic effect through the process of mediating apoptosis $(10,25,26,29)$, we thus hypothesized that MKP1 may protect cells by inactivating JNK. We used both JNK specific inhibitor SP600125 and tamoxifen to treat cells. The results showed that inhibition of JNK activation sharply reduced tamoxifen-induced apoptosis and cleaved PARP expression in MKP1 knockdown MCF7-TR cells and MKP1 low-expressed MCF7 cells, suggesting that JNK signal pathway is indeed involved in the tamoxifen-induced apoptosis, and MKP1 inhibits tamoxifen-induced apoptosis through blocking JNK activation. Furthermore, we used MKP1 inhibitor triptolide in combination with tamoxifen to treat MCF7-TR cells, and found that triptolide promoted tamoxifen-induced activation of JNK and apoptosis in MCF7-TR cells.

In conclusion, we showed that MKP1 expression increased in tamoxifen resistant MCF7-TR cells compared with parental MCF7 cells. We also showed that high expression of MKP1 in MCF7-TR and MCF7 cells can protect cells from tamoxifen-induced apoptosis, and this is dependent on the role MKP1 played in regulation of JNK activation. Moreover, our result suggested that targeting MKP1 may be a potential therapeutic strategy for tamoxifen resistance.

\section{Acknowledgements}

This study was supported by grant from the Science and Technology Research and Development Foundation of Shaanxi Province (no. 2011K13-01-08 and no. 2014KRM99-02). We thank Professor Xinyi Chen (Beijing University of Chinese Medicine) for providing tamoxifen resistant MCF7 cells and Professor Lianfeng Zhang (Peking Union Medical Collage) for providing MKP-1 cDNA plasmid.

\section{References}

1. Cancer Genome Atlas N; Cancer Genome Atlas Network: Comprehensive molecular portraits of human breast tumours. Nature 490: 61-70, 2012.

2. Osborne CK and Schiff R: Mechanisms of endocrine resistance in breast cancer. Annu Rev Med 62: 233-247, 2011.

3. Osborne CK and Osborne CK: Tamoxifen in the treatment of breast cancer. N Engl J Med 339: 1609-1618, 1998.

4. Riggins RB, Schrecengost RS, Guerrero MS and Bouton AH: Pathways to tamoxifen resistance. Cancer Lett 256: 1-24, 2007.

5. García-Becerra R, Santos N, Díaz L and Camacho J: Mechanisms of resistance to endocrine therapy in breast cancer: Focus on signaling pathways, miRNAs and genetically based resistance. Int J Mol Sci 14: 108-145, 2012.

6. Kim EK and Choi EJ: Pathological roles of MAPK signaling pathways in human diseases. Biochim Biophys Acta 1802: 396-405, 2010.

7. Britton DJ, Hutcheson IR, Knowlden JM, Barrow D, Giles M, McClelland RA, Gee JM and Nicholson RI: Bidirectional cross talk between ERalpha and EGFR signalling pathways regulates tamoxifen-resistant growth. Breast Cancer Res Treat 96: 131-146, 2006.

8. Gutierrez MC, Detre S, Johnston S, Mohsin SK, Shou J Allred DC, Schiff R, Osborne CK and Dowsett M: Molecular changes in tamoxifen-resistant breast cancer: Relationship between estrogen receptor, HER-2, and p38 mitogen-activated protein kinase. J Clin Oncol 23: 2469-2476, 2005.

9. Dumont JA, Bitonti AJ, Wallace CD, Baumann RJ, Cashman EA and Cross-Doersen DE: Progression of MCF-7 breast cancer cells to antiestrogen-resistant phenotype is accompanied by elevated levels of AP-1 DNA-binding activity. Cell Growth Differ 7: 351-359, 1996.

10. Mandlekar S, Yu R, Tan TH and Kong AN: Activation of caspase-3 and c-Jun NH2-terminal kinase-1 signaling pathways in tamoxifen-induced apoptosis of human breast cancer cells. Cancer Res 60: 5995-6000, 2000.

11. Theodosiou A and Ashworth A: MAP kinase phosphatases. Genome Biol 3: S3009, 2002.

12. Camps M, Nichols A and Arkinstall S: Dual specificity phosphatases: A gene family for control of MAP kinase function. FASEB J 14: 6-16, 2000.

13. Wu JJ, Roth RJ, Anderson EJ, Hong EG, Lee MK, Choi CS, Neufer PD, Shulman GI, Kim JK and Bennett AM: Mice lacking MAP kinase phosphatase-1 have enhanced MAP kinase activity and resistance to diet-induced obesity. Cell Metab 4: 61-73, 2006.

14. Zhao Q, Wang X, Nelin LD, Yao Y, Matta R, Manson ME, Baliga RS, Meng X, Smith CV, Bauer JA, et al: MAP kinase phosphatase 1 controls innate immune responses and suppresses endotoxic shock. J Exp Med 203: 131-140, 2006.

15. Wancket LM, Frazier WJ and Liu Y: Mitogen-activated protein kinase phosphatase (MKP)-1 in immunology, physiology, and disease. Life Sci 90: 237-248, 2012.

16. Li M, Zhou JY, Ge Y, Matherly LH and Wu GS: The phosphatase MKP1 is a transcriptional target of p53 involved in cell cycle regulation. J Biol Chem 278: 41059-41068, 2003.

17. Sánchez-Pérez I, Martínez-Gomariz M, Williams D, Keyse SM and Perona R: CL100/MKP-1 modulates JNK activation and apoptosis in response to cisplatin. Oncogene 19: 5142-5152, 2000. 
18. Wang Z, Xu J, Zhou JY, Liu Y and Wu GS: Mitogen-activated protein kinase phosphatase-1 is required for cisplatin resistance. Cancer Res 66: 8870-8877, 2006.

19. Small GW, Shi YY, Higgins LS and Orlowski RZ: Mitogenactivated protein kinase phosphatase-1 is a mediator of breast cancer chemoresistance. Cancer Res 67: 4459-4466, 2007.

20. Wang Z, Zhou JY, Kanakapalli D, Buck S, Wu GS and Ravindranath Y: High level of mitogen-activated protein kinase phosphatase-1 expression is associated with cisplatin resistance in osteosarcoma. Pediatr Blood Cancer 51: 754-759, 2008.

21. Brünner N, Frandsen TL, Holst-Hansen C, Bei M, Thompson EW, Wakeling AE, Lippman ME and Clarke R: MCF7/LCC2: A 4-hydroxytamoxifen resistant human breast cancer variant that retains sensitivity to the steroidal antiestrogen ICI 182,780 . Cancer Res 53: 3229-3232, 1993.

22. Takeuchi K, Shin-ya T, Nishio K and Ito F: Mitogen-activated protein kinase phosphatase-1 modulated JNK activation is critical for apoptosis induced by inhibitor of epidermal growth factor receptor-tyrosine kinase. FEBS J 276: 1255-1265, 2009.

23. Mandlekar S and Kong AN: Mechanisms of tamoxifen-induced apoptosis. Apoptosis 6: 469-477, 2001.

24. Bogush T, Dudko E, Bogush E, Polotsky B, Tjulandin S and Davydov M: Tamoxifen non-estrogen receptor mediated molecular targets. Oncol Rev 6: e15, 2012.

25. Madeo A, Vinciguerra M, Lappano R, Galgani M, GasperiCampani A, Maggiolini M and Musti AM: c-Jun activation is required for 4-hydroxytamoxifen-induced cell death in breast cancer cells. Oncogene 29: 978-991, 2010.

26. Wang N, Li Z, Tian F, Feng Y, Huang J, Li C and Xie F: PKCa inhibited apoptosis by decreasing the activity of JNK in MCF-7/ADR cells. Exp Toxicol Pathol 64: 459-464, 2012.

27. Chen P, Li J, Barnes J, Kokkonen GC, Lee JC and Liu Y: Restraint of proinflammatory cytokine biosynthesis by mitogen-activated protein kinase phosphatase-1 in lipopolysaccharide-stimulated macrophages. J Immunol 169: 6408-6416, 2002.
28. Zhao Q, Shepherd EG, Manson ME, Nelin LD, Sorokin A and Liu Y: The role of mitogen-activated protein kinase phosphatase-1 in the response of alveolar macrophages to lipopolysaccharide: Attenuation of proinflammatory cytokine biosynthesis via feedback control of p38. J Biol Chem 280: 8101-8108, 2005.

29. Obrero M, Yu DV and Shapiro DJ: Estrogen receptor-dependent and estrogen receptor-independent pathways for tamoxifen and 4-hydroxytamoxifen-induced programmed cell death. J Biol Chem 277: 45695-45703, 2002.

30. Wang HY, Cheng Z and Malbon CC: Overexpression of mitogenactivated protein kinase phosphatases MKP1, MKP2 in human breast cancer. Cancer Lett 191: 229-237, 2003.

31. Rojo F, González-Navarrete I, Bragado R, Dalmases A, Menéndez S, Cortes-Sempere M, Suárez C, Oliva C, Servitja S, Rodriguez-Fanjul V, et al: Mitogen-activated protein kinase phosphatase-1 in human breast cancer independently predicts prognosis and is repressed by doxorubicin. Clin Cancer Res 15: 3530-3539, 2009

32. Wang J, Zhou JY and Wu GS: ERK-dependent MKP-1-mediated cisplatin resistance in human ovarian cancer cells. Cancer Res 67: 11933-11941, 2007.

33. Rabenoelina F, Semlali A, Duchesne MJ, Freiss G, Pons M and Badia E: Effect of prolonged hydroxytamoxifen treatment of MCF-7 cells on mitogen activated kinase cascade. Int J Cancer 98: 698-706, 2002.

34. Creighton CJ, Hilger AM, Murthy S, Rae JM, Chinnaiyan AM and El-Ashry D: Activation of mitogen-activated protein kinase in estrogen receptor alpha-positive breast cancer cells in vitro induces an in vivo molecular phenotype of estrogen receptor alpha-negative human breast tumors. Cancer Res 66: 3903-3911, 2006. 